\title{
Stellar parameters through high precision parallaxes
}

\author{
C. Jordi, C. Fabricius, J. M. Carrasco, F. Figueras, E. Masana, \\ H. Voss and X. Luri \\ Departament d'Astronomia i Meteorologia. Universitat de Barcelona-IEEC \\ Martí Franqués 1, E-08028 Barcelona, Spain, email: carme@am.ub.es
}

\begin{abstract}
Before Hipparcos, the determination of absolute luminosity was usually done through calibrations based on a few stellar parallaxes measured at the highest precision. The Hipparcos mission meant a giant step on the knowledge of luminosities and fine structure of the HR diagram. The Gaia mission will go an enormous step further. Besides luminosities, Gaia will allow us to derive other stellar parameters like temperature and extinction, gravity, chemical composition, age and mass by the combination of astrometric and spectrometric data. Through simulations during the mission preparation, it has been shown that the astrometric parallax information is essential to deal with the degeneracy between gravity and chemical composition $([\mathrm{Fe} / \mathrm{H}]$ and $[\alpha / \mathrm{Fe}])$, that cannot be treated using only spectrophotometry. We show the expected HR diagram for the Gaia domain and the accuracies of stellar parameters.
\end{abstract}

Keywords. stars: fundamental parameters, stars: distances, stars: variables, HertzsprungRussell diagram, techniques: photometric, space vehicles

\section{Introduction}

Gaia is an all-sky survey satellite (Perryman et al. (2001), Lindegren et al. (this volume)), to be launched by ESA around 2012, to obtain parallaxes and proper motions to microarcsecond precision, radial velocities and astrophysical parameters for about $10^{9}$ objects down to a limiting magnitude of $\sim 20$ mag. A 3D reconstruction of the Galactic velocity field, coupled with the physical properties of the stars, will provide answers to long-standing questions about the Galaxy such as its structure, formation, dynamical and chemical evolution, the halo stream properties, and others.

The derivation of the stellar parameters (luminosity, $T_{\text {eff }}, \log g,[\mathrm{Fe} / \mathrm{H}]$, etc.) will mainly be done by combining the low resolution spectra (LR), the white light photometry $(G$ band) and the trigonometric parallax. Figure 1 shows two examples of the variation of the $1 \mathrm{D}$ low resolution red spectra with stellar parameters. For bright stars, the high resolution spectra will allow chemical composition determination as well.

\section{Stellar parameters}

From the measurements of unfiltered (white) light, Gaia will provide magnitudes in a very broad band $(G, 330-1000 \mathrm{~nm})$, with estimated uncertainties are shown in Fig. 2 (left). Figure 2 (right) shows the stars with parallaxes better than $10 \%$ in a simulated HR diagram given the instrument model and the Besançon Galaxy model (Robin et al. 2004). The 1 mmag uncertainty at $V \sim 18.5-19.0$ coupled with high precision parallax will allow to calibrate the absolute luminosities of all types of stars across the HR diagram.

The stellar parameters yield the spectral shape and features of the blue and red LR spectra. However, the variation of the shape and features may be caused by variations in 

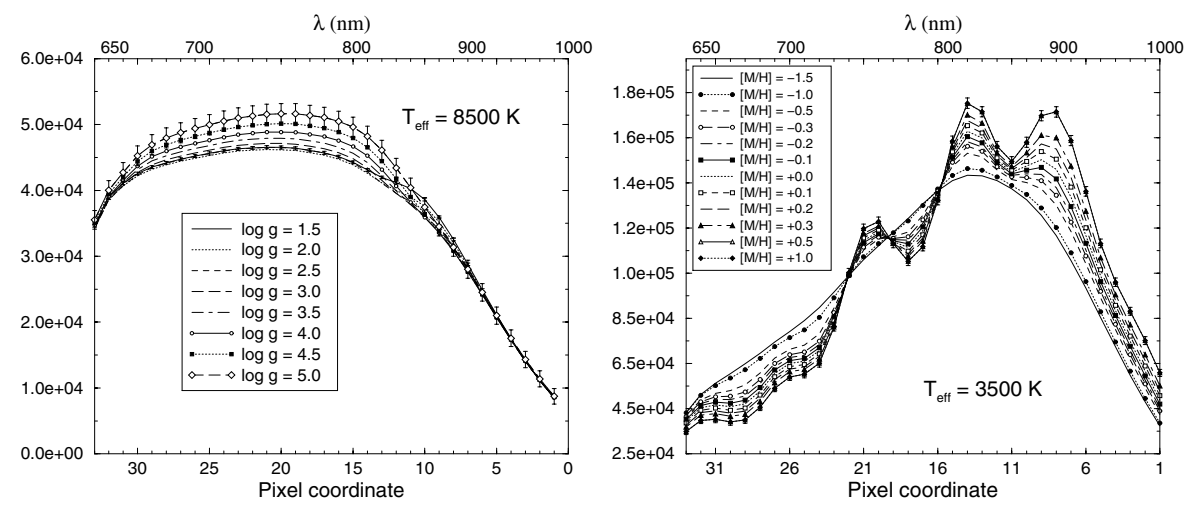

Figure 1. 1D-Red low resolution spectra for several $\log g$ (left) and for several $[\mathrm{Fe} / \mathrm{H}]$ (right) for $G=15$ stars. The bars show the end-of-mission errors for $G=18$.
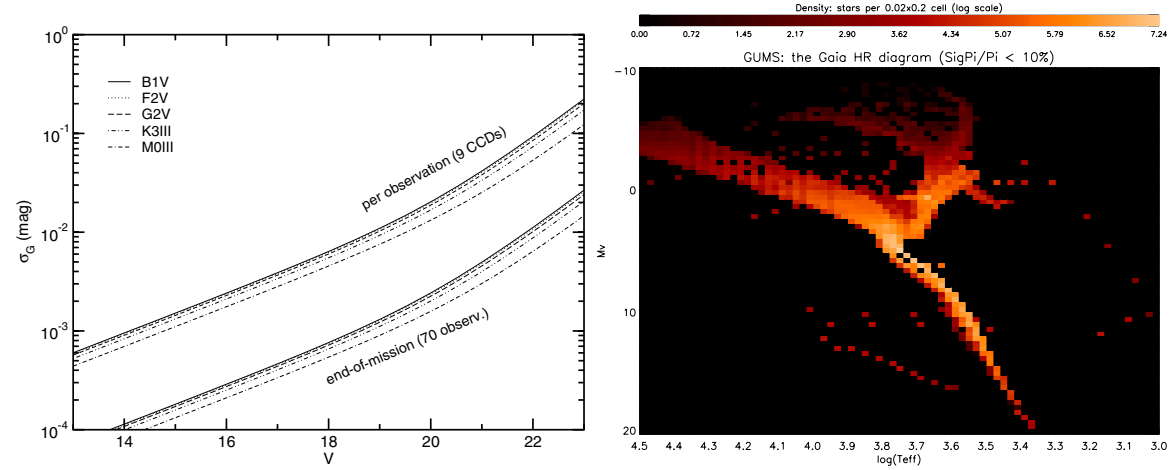

Figure 2. Left: Per transit and the end-of-mission estimated uncertainties of the white light $G$ measurements (calibration errors of about 1 mmag not included). Right: Simulated HR diagram showing the stars with trigonometric parallaxes better than $10 \%$ (about $10^{8}$ stars). The Besançon Galaxy model has been used to represent the stellar content of the Galaxy.

more than one stellar parameter. In the case of Gaia, the extremely precise parallaxes will allow to handle such degeneracies in many cases (for instance, $[\mathrm{Fe} / \mathrm{H}],[\alpha / \mathrm{Fe}]$ and $\log g$ degeneracy). The uncertainties of stellar parameters taking into account the parallax information have been estimated. The results show that individual ages for halo stars can be derived with a precision of 1.5 and $2.2 \mathrm{Gyr}$ at 2.5 and $5 \mathrm{kpc}$, respectively. For halo $\mathrm{G}$ and $\mathrm{K}$ giants, the $[\mathrm{Fe} / \mathrm{H}]$ values will be precise to $0.07,0.15$ and 0.20 dex at 5,12 and $17 \mathrm{kpc}$, respectively. For disk stars, the ages will have precision of 1.2 and $4.7 \mathrm{Gyr}$ at 2 and $3 \mathrm{kpc}$, respectively towards the galactic center, and 1.1 and $2.6 \mathrm{Gyr}$ at the same distances in the anticenter direction. The $[\mathrm{Fe} / \mathrm{H}]$ precisions will be of 0.05 and $0.20 \mathrm{dex}$ at 2 and $5 \mathrm{kpc}$, respectively towards the center and $0.05,0.10$ and 0.15 at 2, 5, and 8 kpc, towards the anticenter.

Acknowledgements: This project is supported by the Spanish MEC under contract PNE200613855-C02-01.

\section{References}

Perryman, M. A. C., de Boer, K. S., Gilmore, G., et al., 2001, A\&A, 369, 339

Robin, A. C., Reylé, C., Derriére, S., \& Picaud, S. 2004, A\&A, 409, 523 